\title{
A Method for Generating Approximate Similarity Solutions of Nonlinear Partial Differential Equations
}

\author{
Mazhar Iqbal, ${ }^{1}$ M. T. Mustafa, ${ }^{2}$ and Azad A. Siddiqui ${ }^{3}$ \\ ${ }^{1}$ Department of Basic Sciences and Humanities, EME College, National University of Sciences and Technology (NUST), \\ Peshawar Road, Rawalpindi 46000, Pakistan \\ ${ }^{2}$ Department of Mathematics, Statistics and Physics, Qatar University, P.O. Box 2713, Doha, Qatar \\ ${ }^{3}$ School of Natural Sciences (SNS), National University of Sciences and Technology (NUST), Sector H-12, \\ Islamabad 44000, Pakistan \\ Correspondence should be addressed to Mazhar Iqbal; drmazhar@ceme.nust.edu.pk
}

Received 24 April 2014; Revised 7 July 2014; Accepted 8 July 2014; Published 22 July 2014

Academic Editor: Bashir Ahmad

Copyright (C) 2014 Mazhar Iqbal et al. This is an open access article distributed under the Creative Commons Attribution License, which permits unrestricted use, distribution, and reproduction in any medium, provided the original work is properly cited.

\begin{abstract}
Standard application of similarity method to find solutions of PDEs mostly results in reduction to ODEs which are not easily integrable in terms of elementary or tabulated functions. Such situations usually demand solving reduced ODEs numerically. However, there are no systematic procedures available to utilize these numerical solutions of reduced ODE to obtain the solution of original PDE. A practical and tractable approach is proposed to deal with such situations and is applied to obtain approximate similarity solutions to different cases of an initial-boundary value problem of unsteady gas flow through a semi-infinite porous medium.
\end{abstract}

\section{Introduction}

The mathematical modeling of most of the physical processes in fields like diffusion, chemical kinetics, fluid mechanics, wave mechanics, and general transport problems is governed by such nonlinear PDEs whose analytic solutions are hard to find. Therefore, the approach of investigating nonlinear PDEs via reduction to ODEs becomes important and has been quite fruitful in analysis of many physical problems. The reader is referred to [1] for an introduction to different types of such reduction approaches and to have an idea about the advances made in the fields of nonlinear diffusion, fluid mechanics, and wave propagation from the utilization of reduction-to-ODE approach.

Lie symmetry method provides a powerful general technique for analyzing nonlinear PDEs and reducing them to ODEs. PDEs modeling physical processes naturally inherit symmetries from the underlying physical system. Lie symmetry method takes advantage of these natural symmetries in a PDE and provides similarity variables that lead to the reduction to ODEs; compare, for example, [2-4]. A large amount of the literature about the Lie symmetry theory, its applications, and its extensions is available; see, for example, $[1,5-12]$.

Although there have been some notable contributions in the applications of similarity method to boundary value problems of PDEs (cf. [1, 12-15]), in general the method has not been utilized in a great deal mainly because of a restriction explained below. The success of the method relies heavily on the success in solving the reduced ODE. Only in few cases, the reduced ODE is integrable in terms of elementary or tabulated functions, but in most cases it is not a simple matter and it is suggested to resort to numerical methods to solve the reduced ODE. This retreat from analytic calculations to numerical computations is not uncommon, but it has not proved practical due to the lack of practical and systematic procedures to utilize the numerical solution of reduced ODE to obtain the solution of original PDE. The aim of this work is to provide one practical way of dealing with such situations.

As a test problem to demonstrate our method, we consider a physical problem arising in the transient flow of gas 
through a semi-infinite porous medium. In the investigation of the unsteady flow of gas through a semi-infinite porous medium [16-19] initially filled with gas at a uniform pressure $p_{0}>0$ at time $t=0$, the pressure at the outflow face is suddenly reduced from $p_{0}$ to $p_{1} \geq 0\left(p_{1}=0\right.$ is the case of diffusion into a vacuum) and is thereafter maintained at this lower pressure. The studies in $[18,20]$ show that the unsteady flow of gas in a porous medium is modeled by a nonlinear partial differential equation.

The nonlinear partial differential equation that describes the unsteady flow of gas through a semi-infinite porous medium has been derived by Muskat [20] in the form

$$
\nabla^{2}\left(p^{2}\right)=2\left(\frac{\phi \mu}{k}\right) \frac{\partial p}{\partial t}
$$

where $p$ is the pressure within porous medium, $\phi$ is the porosity, $\mu$ is the viscosity, and $k$ is the permeability. In the one-dimensional medium extending from $x=0$ to $x=\infty$, this reduces to

$$
\frac{\partial}{\partial x}\left(p \frac{\partial p}{\partial x}\right)=A \frac{\partial p}{\partial t}
$$

with the boundary conditions

$$
\begin{gathered}
p(x, 0)=p_{0}, \quad 0<x<\infty \\
p(0, t)=p_{1}\left(<p_{0}\right), \quad 0 \leq t<\infty,
\end{gathered}
$$

where the constant $A=\phi \mu / k$ is given by the properties of the medium.

In the next section we provide details about the method and its application to obtain approximate similarity solutions of BVPs of the above form.

\section{The Method and Its Implementation}

The main idea of the method rests on finding an approximation of solution of reduced ODE in the form of a function. This has clear advantages over the option of working only with numerical solution of reduced ODE. In the first place, it can be used to generate approximate solution of original PDE via the similarity variables. Secondly, having the approximate solution of PDE in function form can be more useful than numerical solution as it displays the parameters and variables of the problem, so it requires less processing time and can be used for applications in real time. A detailed description of the method is provided below. IBVP

To explain and illustrate our method we consider the

$$
\frac{\partial}{\partial x}\left(p \frac{\partial p}{\partial x}\right)=A \frac{\partial p}{\partial t}
$$

with

$$
p(x, 0)=p_{0}, \quad p(0, t)=p_{1}\left(<p_{0}\right), \quad p(\infty, t)=p_{0} .
$$

Without loss of generality we can assume $p_{0}=1$ because the change of variable $\bar{p}(x, t)=p(x, t) / p_{0}$ leads to a similar problem with $\bar{p}(x, 0)=1$. So the IBVP under study here is

$$
\frac{\partial}{\partial x}\left(p \frac{\partial p}{\partial x}\right)=A \frac{\partial p}{\partial t}
$$

with initial and boundary conditions

$$
\begin{gathered}
p(x, 0)=1, \quad 0<x<\infty, \\
p(0, t)=p_{1}(<1), \quad 0 \leq t<\infty, \\
p(\infty, t)=1, \quad 0<t<\infty .
\end{gathered}
$$

Step 1 (Reduction of IVBP to a BVP of ODE). The similarity transformations $[16,17]$

$$
z=\frac{x}{\sqrt{t}}\left(\frac{A}{4}\right)^{1 / 2}, \quad w(z)=\alpha^{-1}\left(1-(p(x, t))^{2}\right)
$$

with $\alpha=1-p_{1}^{2}$, reduce the above IBVP (6)-(7) to BVP of ODE as follows:

$$
\begin{gathered}
w^{\prime \prime}+\frac{2 z}{\sqrt{1-\alpha w}} w^{\prime}=0, \\
w(z=0)=1, \quad w(z \longrightarrow \infty)=0 .
\end{gathered}
$$

The aim of the remaining steps is to find an approximate solution $W_{\text {Approx }}(z)$ of BVP (9) in function form and then use the similarity transformations (8) to obtain approximate $p(x, t)$ of PDE problem (6)-(7).

Step 2. Find numerical solution $W_{\text {Num }}$ of BVP (9) and use this as a benchmark for obtaining function form $W_{\text {Approx }}$ of the solution.

Step 3 (Obtain an initial guess for $W_{\text {Approx }}$ ). This is a crucial step and in our case we use the lower solution of BVP (9), obtained in [17], as our initial guess. As shown in numerical simulations below, lower solution provides a good initial guess that leads to an accurate enough approximate solution in few iterations. So the initial guess for approximate solution of BVP (9) for all the cases below is taken as follows, see [17, Example 2.1]:

$$
\text { initial approximation }=W_{\text {Lower }}=1-\operatorname{erf}\left(\frac{z}{\sqrt{p_{1}}}\right) \text {, }
$$

where erf denotes the error function.

As an alternate, a solution obtained by homotopy analysis method can also be used as initial guess.

Step 4. Improve the initial approximation to get $W_{\text {Approx }}$ up to the desired level of accuracy.

Here we adopt the following procedure for improving the level of accuracy, starting from initial approximation. The lower solution is of the form

$$
1-\operatorname{erf}(k z)
$$


with $k=k_{0}=1 / \sqrt{p_{1}}$ giving the initial approximation $W_{k_{0}}$, that is, the lower solution. Numerical simulations suggest that as the values of $k$ decrease from $k_{0}$ by a small decrement, the lower solution moves uniformly towards the numerical solution.

Given a function $N(x)$ and a number $\varepsilon>0$, we say that $f(x)$ lies within $\varepsilon$-band of $N(x)$ on an interval $I$ if

$$
|f(x)-N(x)|<\varepsilon, \quad \forall x \in I .
$$

For a suitable value $n$ and numbers $\varepsilon>0, \delta_{i}>0$, using the sequences of values

$$
k=k_{i}=k_{0}-\delta_{i}, \quad(i=1,2, \ldots, n),
$$

in (11) generates a sequence of curves $W_{k_{i}}$ that uniformly approach numerical solution, finally resulting in the curve

$$
W_{\text {Approx }}=W_{k_{n}},
$$

which lies in an $\varepsilon$-band around the graph of numerical solution $W_{\text {Num }}$. The number $\varepsilon$ is chosen according to the desired level of accuracy and the value of $k_{n}$ is approximated via numerical simulations.

Step 5. Use the similarity variables (8) to get the approximate solution of original IBVP (6)-(7).

In the subsequent subsections we illustrate implementation of the above procedure, and we provide simulation results and approximate similarity solutions for different cases of values of parameter $p_{1}$.

2.1. Approximate Similarity Solution of the IBVP (6)-(7) for $p_{1}=0.9$. In this case, $k=1 / \sqrt{0.9}$ and the lower solution of the ODE problem (9) becomes

$$
\begin{aligned}
W_{\text {Lower }} & =1-\operatorname{erf}(k z)=1-\operatorname{erf}\left(\frac{z}{\sqrt{0.9}}\right) \\
& \approx 1-\operatorname{erf}(1.05409 z),
\end{aligned}
$$

which serves as our initial approximation $W_{k_{0}}$ for approximating the solution of ODE problem. Solving the BVP (9) numerically to get $W_{\text {Num }}$ and uniformly improving the approximations $W_{k_{i}}$ by simulating the procedure explained above we obtain an approximate solution of the ODE problem as

$$
W_{\text {Approx }}(z)=1-\operatorname{erf}(0.7281 z)
$$

with

$$
\operatorname{Max}\left|W_{\text {Approx }}-W_{\text {Num }}\right|=0.0019642487 .
$$

The plots of the initial approximation $W_{\text {Lower }}(z)$, the numerical solution $W_{\text {Num }}(z)$, the approximate solution $W_{\text {Approx }}(z)$, and the $\operatorname{Error}(z)$ are given in Figure 1 where

$$
\operatorname{Error}(z)=W_{\text {Approx }}(z)-W_{\text {Num }}(z) .
$$

The dotted curves demonstrate some intermediary curves involved in simulations of the uniform approximation process from $W_{\text {Lower }}$ to $W_{\text {Approx }}$, in a manner that as $k$ decreases

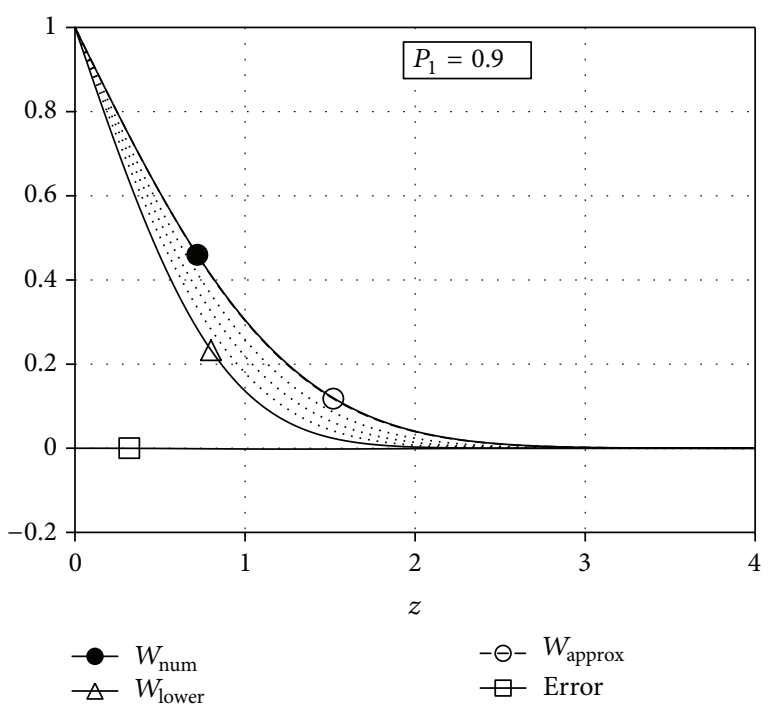

Figure 1

from $1 / \sqrt{0.9}$ by a small decrement the curves $1-\operatorname{erf}(k z)$ move uniformly towards numerical solution $W_{\text {Num }}$.

Finally the similarity variables (8) provide the approximate solution of IBVP (6)-(7) for $p_{1}=0.9$ as

$$
p(x, t)=\sqrt{0.81+0.19 \operatorname{erf}\left(0.7281 \frac{x}{\sqrt{t}}\left(\frac{A}{4}\right)^{1 / 2}\right)} .
$$

2.2. Approximate Similarity Solution of the IBVP (6)-(7) for $p_{1}=0.3$. In this case, $k=1 / \sqrt{0.3}$ and hence the initial approximation determined by the lower solution of the ODE problem (9) becomes

$$
\begin{aligned}
W_{\text {Lower }} & =1-\operatorname{erf}(k z)=1-\operatorname{erf}\left(\frac{z}{\sqrt{0.3}}\right) \\
& \approx 1-\operatorname{erf}(1.82574 z) .
\end{aligned}
$$

Proceeding as in Section 2.1 we obtain an approximate solution of the ODE problem, for the case $p_{1}=0.3$, as

$$
W_{\text {Approx }}(z)=1-\operatorname{erf}(0.77362 z) \text {, }
$$

with

$$
\operatorname{Max}\left|W_{\text {Approx }}-W_{\text {Num }}\right|=0.0096829849 .
$$

The plots of the initial approximation $W_{\text {Lower }}(z)$, the numerical solution $W_{\text {Num }}(z)$, the approximate solution $W_{\text {Approx }}(z)$, and the $\operatorname{Error}(z)$, for the case $p_{1}=0.3$, are given in Figure 2 .

As in Section 2.1 the dotted curves demonstrate some intermediary curves involved in simulations of the uniform approximation process from $W_{\text {Lower }}$ to $W_{\text {Approx }}$, in a manner that as $k$ decreases from $1 / \sqrt{0.3}$ by a small decrement the curves $1-\operatorname{erf}(k z)$ move uniformly towards numerical solution $W_{\text {Num }}$. 


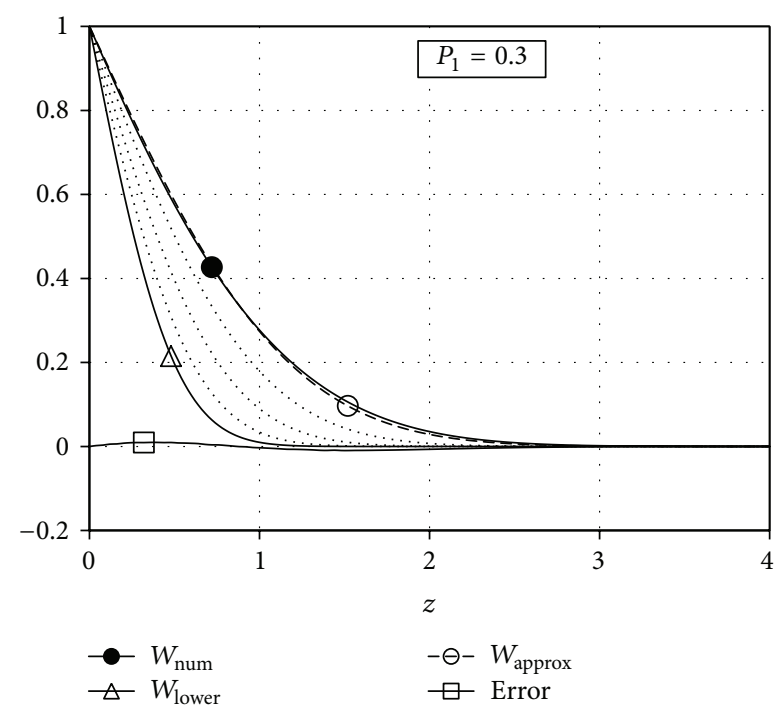

Figure 2

The approximate solution of IBVP (6)-(7) for $p_{1}=0.3 \mathrm{can}$ be found using the similarity variables ( 8 ) and is given by

$$
p(x, t)=\sqrt{0.09+0.91 \operatorname{erf}\left(0.77362 \frac{x}{\sqrt{t}}\left(\frac{A}{4}\right)^{1 / 2}\right)} .
$$

2.3. Approximate Similarity Solution of the IBVP (6)-(7) for $p_{1}=0.1$. Using $k=1 / \sqrt{0.1}$ gives the lower solution of the ODE problem (9) as

$$
\begin{aligned}
W_{\text {Lower }} & =1-\operatorname{erf}(k z)=1-\operatorname{erf}\left(\frac{z}{\sqrt{0.1}}\right) \\
& \approx 1-\operatorname{erf}(3.162277 z),
\end{aligned}
$$

which serves as our initial approximation $W_{k_{0}}$ for approximating the solution of ODE problem for this case. As above, solving the BVP (9) numerically to get $W_{\text {Num }}$ and uniformly improving the approximations $W_{k_{i}}$ by simulating the procedure explained above, we obtain an approximate solution of the ODE problem as

$$
W_{\text {Approx }}(z)=1-\operatorname{erf}(0.782743 z),
$$

with

$$
\operatorname{Max}\left|W_{\text {Approx }}-W_{\text {Num }}\right|=0.0116428185 .
$$

The plots of the initial approximation $W_{\text {Lower }}(z)$, the numerical solution $W_{\text {Num }}(z)$, the approximate solution $W_{\text {Approx }}(z)$, and the $\operatorname{Error}(z)$, for the case $p_{1}=0.1$, are given in Figure 3. As in the above sections, the dotted curves demonstrate some intermediary curves involved in simulations of the uniform approximation process from $W_{\text {Lower }}$ to $W_{\text {Approx }}$, in a manner that as $k$ decreases from $1 / \sqrt{0.1}$ by a small decrement the curves $1-\operatorname{erf}(k z)$ move uniformly towards numerical solution $W_{\text {Num }}$.

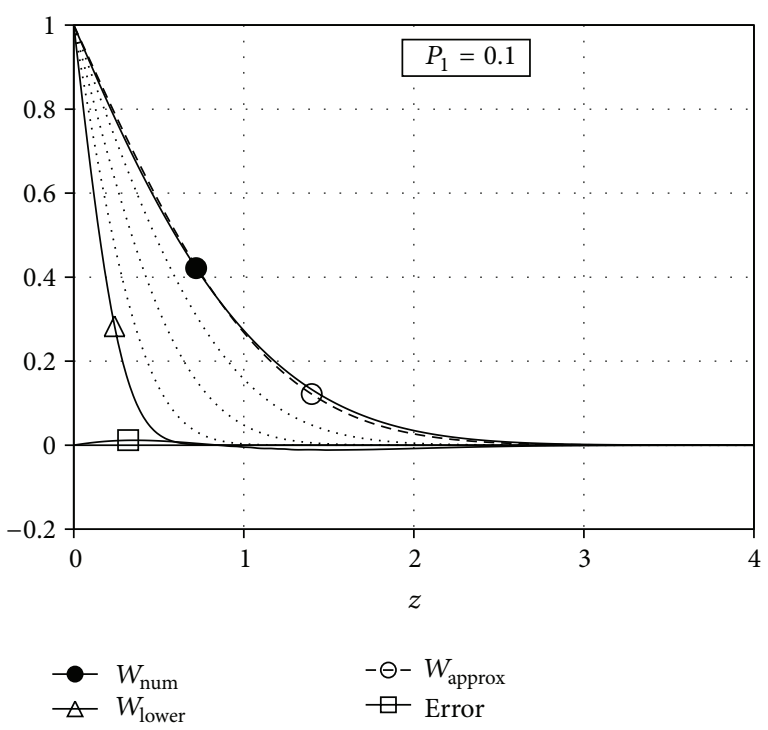

Figure 3

Using the similarity variables, the approximate solution of IBVP (6)-(7) for $p_{1}=0.1$ is found as

$$
p(x, t)=\sqrt{0.01+0.99 \mathrm{erf}\left(0.782743 \frac{x}{\sqrt{t}}\left(\frac{A}{4}\right)^{1 / 2}\right)} .
$$

2.4. Discussion. The plots of the numerical solution $W_{\mathrm{Num}}(z)$, the lower solution $W_{\text {Lower }}(z)$, and the approximate solution $W_{\text {Approx }}(z)$ for the ODE problem (9) are given in Figures 1, 2 , and 3 , respectively, for $p_{1}=0.9, p_{1}=0.3$, and $p_{1}=0.1$. It can be seen that the lower solution $W_{\text {Lower }}(z)$ provides a better initial approximation for the values of $p_{1}$ closer to 1. Yet a comparison of the plots of initial approximation $W_{\text {Lower }}(z)$ and the accuracy benchmark curve $W_{\text {Num }}(z)$, in all cases, clearly emphasizes the need of improving the initial approximation to get $W_{\text {Lower }}(z)$ to get accurate enough analytic approximation $W_{\text {Approx }}(z)$ of the benchmark curve $W_{\text {Num }}(z)$. The plots in Figures 1,2 , and 3 clearly show that $W_{\text {Approx }}(z)$ and $W_{\text {Num }}(z)$ curves overlap in each case and the corresponding plots of the error $\operatorname{Error}(z)$ demonstrate that, in each case, $W_{\text {Approx }}(z)$ provides an analytic approximation of the benchmark numerical solution curve $W_{\text {Num }}(z)$. In all cases, the solution $W_{\text {Approx }}(z)$ decreases with the increase in $z$ but descends faster for the case $p_{1}=0.1$ as compared to case $p_{1}=0.9$.

A comparison of the approximate solution $W_{\text {Approx }}(z)$ of BVP (9) obtained by our method with solutions obtained by other methods [21-23], for $\alpha=0.5$, is presented in Table 1 .

2.5. Comments on the Conservation Laws. The conservation laws of

$$
\frac{\partial}{\partial x}\left(p \frac{\partial p}{\partial x}\right)=\frac{\partial p}{\partial t}
$$

can be found using the direct multiplier method [24, 25]. Since the procedure is standard, only the results are stated. 
TABLE 1: Solution values for $\alpha=0.5$.

\begin{tabular}{cccc}
\hline$z$ & $W_{\text {Approx }}(z)$ & $\begin{array}{c}\text { Corresponding solution } \\
W_{\text {kidder }}[18]\end{array}$ & $\begin{array}{c}\text { Corresponding solution } \\
W_{[3 / 3]}[21-23]\end{array}$ \\
\hline 0.1 & 0.899431 & 0.881659 & 0.897917 \\
0.2 & 0.800454 & 0.766308 & 0.798523 \\
0.3 & 0.704584 & 0.656538 & 0.704113 \\
0.4 & 0.613194 & 0.554402 & 0.616504 \\
0.5 & 0.527452 & 0.461365 & 0.537053 \\
0.6 & 0.448284 & 0.378311 & 0.466563 \\
0.7 & 0.37634 & 0.305598 & 0.406243 \\
0.8 & 0.311998 & 0.243133 & 0.35608 \\
0.9 & 0.255363 & 0.190462 & 0.317997 \\
1.0 & 0.206302 & 0.158769 & 0.290026 \\
\hline
\end{tabular}

Implementing the direct multiplier procedure for multipliers of the form $\Lambda\left(t, x, p, p_{x}, p_{x x}\right)$ generates the determining equations

$$
\begin{gathered}
\Lambda_{x x}=0, \quad \Lambda_{t}=0, \quad \Lambda_{p}=0, \\
\Lambda_{p_{x}}=0, \quad \Lambda_{p_{x x}}=0,
\end{gathered}
$$

which yield the solution $\Lambda=C_{1}+C_{2} x$. Hence there are two linearly independent conservation laws arising from the multipliers $\Lambda_{1}=1$ and $\Lambda_{2}=x$. Next, elementary direct calculations [26] give the corresponding conservation laws as listed below.

For $\Lambda_{1}=1$, the conservation law is

$$
D_{t}(p)+D_{x}\left(-p p_{x}\right)=0
$$

and, for $\Lambda_{2}=x$, the conservation law is

$$
D_{t}(x p)+D_{x}\left(\frac{p^{2}}{2}-x p p_{x}\right)=0,
$$

where

$$
\begin{aligned}
& D_{t}=\frac{\partial}{\partial t}+p_{t} \frac{\partial}{\partial p}+p_{t t} \frac{\partial}{\partial p_{t}}+p_{t x} \frac{\partial}{\partial p_{x}}+\cdots \\
& D_{x}=\frac{\partial}{\partial x}+p_{x} \frac{\partial}{\partial p}+p_{x t} \frac{\partial}{\partial p_{t}}+p_{x x} \frac{\partial}{\partial p_{x}}+\cdots
\end{aligned}
$$

For a given conservation law

$$
D_{t}\left(T^{1}\right)+D_{x}\left(T^{2}\right)=0,
$$

of (28), if the spatial flux $T^{2}$ vanishes on the boundary $x=0$ and $x=\infty$ of the semi-infinite medium, then integration from $x=0$ to $x=\infty$ provides conserved quantity of the boundary value problem.

As an example, the conservation law (30) is applied to derive conserved quantity of the BVP

$$
\begin{gathered}
\frac{\partial}{\partial x}\left(p \frac{\partial p}{\partial x}\right)=\frac{\partial p}{\partial t} \\
\frac{\partial p}{\partial x}(0, t)=0, \quad \frac{\partial p}{\partial x} \longrightarrow k e^{-x^{2} / t}, \quad \text { as } x \longrightarrow \infty .
\end{gathered}
$$

The conservation law (30) can be written as

$$
\frac{\partial}{\partial t}(p)-\frac{\partial}{\partial x}\left(p p_{x}\right)=0
$$

Integrating with respect to $x$ from $x=0$ to $x=\infty$ and using the boundary conditions imply that

$$
\frac{\partial}{\partial t} \int_{0}^{\infty} p(x, t) d x=0
$$

which gives the time independent conserved quantity $\int_{0}^{\infty} p(x, t) d x$ of the BVP.

In general, the boundary conditions will determine which conservation law is to be applied to obtain conserved quantities of the BVP of (28).

\section{Conclusion}

We present a practical way of obtaining approximate solution, in function form, for the class of PDEs where the PDE can be reduced to an ODE through similarity variables but the reduced ODE is not easily integrable in terms of elementary or tabulated functions. The idea presented here to get approximate solution of PDE, that is, approximating the surface $p(x, t)$, practically involves approximating a curve $W(z)$ which is a tractable problem in comparison to increasingly complex and intractable problem of approximating the surface $p(x, t)$ itself. A combination of simulations, initial approximation, and numerical solution of reduced ODE is utilized to obtain approximation of solution curve $W(z)$ which readily generates, via similarity variables, the approximate solution surface $p(x, t)$ of the PDE. This makes it a promising approach especially when reasonably accurate initial approximation of the solution of ODE can be obtained, as was the case here in the form of lower solution. The approach is applied to obtain approximate solutions for some cases of an initial-boundary value problem of unsteady flow of gas through a semi-infinite porous medium. The approach can be adapted for obtaining approximate analytic solutions for the class of PDEs where the PDE can be reduced to an ODE through similarity variables. For instance, the approach can be directly applied to all the reduced-via-similarity BVPs of ODEs in [27]. For further application, the approach can be extended to obtain approximate solutions where the reduction is a system of ODEs like the reduced flow problems in $[28,29]$.

\section{Conflict of Interests}

The authors declare that there is no conflict of interests regarding the publication of this paper.

\section{References}

[1] W. F. Ames, Nonlinear Partial Differential Equations in Engineering, vol. I, Academic Press, New York, NY, USA, 1965.

[2] H. Azad, M. T. Mustafa, and M. Ziad, "Group classification, optimal system and optimal reductions of a class of Klein Gordon equations," Communications in Nonlinear Science and Numerical Simulation, vol. 15, no. 5, pp. 1132-1147, 2010. 
[3] A. H. Bokhari, M. T. Mustafa, and F. D. Zaman, "An exact solution of a quasilinear Fisher equation in cylindrical coordinates," Nonlinear Analysis, vol. 69, no. 12, pp. 4803-4805, 2008.

[4] M. T. Mustafa and K. Masood, "Symmetry solutions of a nonlinear elastic wave equation with third-order anharmonic corrections," Applied Mathematics and Mechanics, vol. 30, no. 8, pp. 1017-1026, 2009.

[5] G. W. Bluman and J. D. Cole, Similarity Methods for Differential Equations, Springer, New York, NY, USA, 1974.

[6] G. W. Bluman and S. Kumei, Symmetries and Differential Equations, Springer, New York, NY, USA, 1989.

[7] A. G. Hansen, Similarity Analyses of Boundary Value Problems in Engineering, Prentice Hall, Englewood Cliffs, NJ, USA, 1964.

[8] P. E. Hydon, Symmetry Methods for Differential Equations, Cambridge University Press, Cambridge, UK, 2000.

[9] N. H. Ibragimov, Elementary Lie Group Analysis and Ordinary Differential Equations, John Wiley \& Sons, Chichester, UK, 1999.

[10] W. Miller, Symmetry and Separation of Variables, AddisonWesley, Reading, Mass, USA, 1977.

[11] P. J. Olver, Applications of Lie Groups to Differential Equations, Springer, New York, NY, USA, 1986.

[12] L. V. Ovsiannikov, Group Analysis of Differential Equations, Academic Press, New York, NY, USA, 1982.

[13] H. Azad, M. T. Mustafa, and A. F. M. Arif, "Analytic solutions of initial-boundary-value problems of transient conduction using symmetries," Applied Mathematics and Computation, vol. 215, no. 12, pp. 4132-4140, 2010.

[14] G. W. Bluman, "Applications of the general similarity solution of the heat equation to boundary-value problems," Quarterly of Applied Mathematics, vol. 31, pp. 403-415, 1974.

[15] B. J. Cantwell, Introduction to Symmetry Analysis, Cambridge University Press, Cambridge, UK, 2002.

[16] R. P. Agarwal and D. O’Regan, "Infinite interval problems modeling the flow of a gas through a semi-infinite porous medium," Studies in Applied Mathematics, vol. 108, no. 3, pp. 245-257, 2002.

[17] R. P. Agarwal and D. O’Regan, "Non-linear boundary value problems on the semi-infinite interval: an upper and lower solution approach," Mathematika, vol. 49, no. 1-2, pp. 129-140, 2002.

[18] R. E. Kidder, "Unsteady flow of gas through a semi-infinite porous medium," Journal of Applied Mechanics, vol. 27, pp. 329332, 1957.

[19] T. Y. Na, Computational Methods in Engineering Boundary Value Problems, vol. 145 of Mathematics in Science and Engineering, Academic Press, New York, NY. USA, 1979.

[20] M. Muskat, Flow of Homogeneous Fluids, J. Edwards, Ann Arbor, Mich, USA, 1946.

[21] Y. Khan, N. Faraz, and A. Yildirim, "Series solution for unsteady gas equation via Mldm-Pade Technique," World Applied Sciences Journal, vol. 10, pp. 1452-1456, 2010.

[22] M. A. Noor and S. T. Mohyud-Din, "Variational iteration method for unsteady flow of gas through a porous medium using He's polynomials and Pade approximants," Computers \& Mathematics with Applications, vol. 58, no. 11-12, pp. 2182-2189, 2009.

[23] A. Wazwaz, "The modified decomposition method applied to unsteady flow of gas through a porous medium," Applied Mathematics and Computation, vol. 118, no. 2-3, pp. 123-132, 2001.
[24] S. C. Anco and G. Bluman, "Direct constructi on method for conservation laws of partial di ff erential equations part I: examples of c onservation law classifications," European Journal of Applied Mathematics, vol. 13, no. 5, pp. 545-566, 2002.

[25] S. C. Anco and G. Bluman, "Direct construction method for conservation laws of partial differential equations part II: general treatment," European Journal of Applied Mathematics, vol. 13, no. 5, pp. 567-585, 2002.

[26] T. Wolf, "A comparison of four approaches to the calculation of conservation laws," European Journal of Applied Mathematics, vol. 13, no. 2, pp. 129-152, 2002.

[27] L. Dresner, Similarity Solutions of Nonl inear Partial Differential Equations, vol. 88 of Research Notes in Mathematics Series, Longman, 1983.

[28] M. M. Rashidi, T. Hayat, E. Erfani, S. A. M. Pour, and A. A. Hendi, "Simultaneous effects of partial slip and thermaldiffusion and diffusion-thermo on steady MHD convective flow due to a rotating disk," Communications in Nonlinear Science and Numerical Simulation, vol. 16, no. 11, pp. 4303-4317, 2011.

[29] M. M. Rashidi, S. A. Mohimanian Pour, T. Hayat, and S. Obaidat, "Analytic approximate solutions for steady flow over a rotating disk in porous medium with heat transfer by homotopy analysis method," Computers \& Fluids, vol. 54, no. 1, pp. 1-9, 2012. 


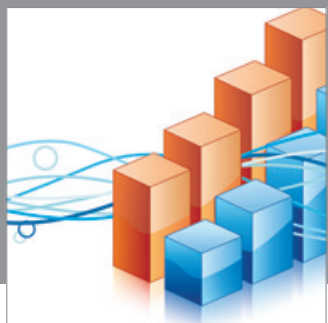

Advances in

Operations Research

mansans

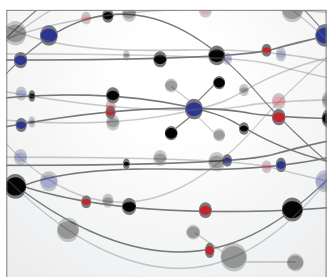

The Scientific World Journal
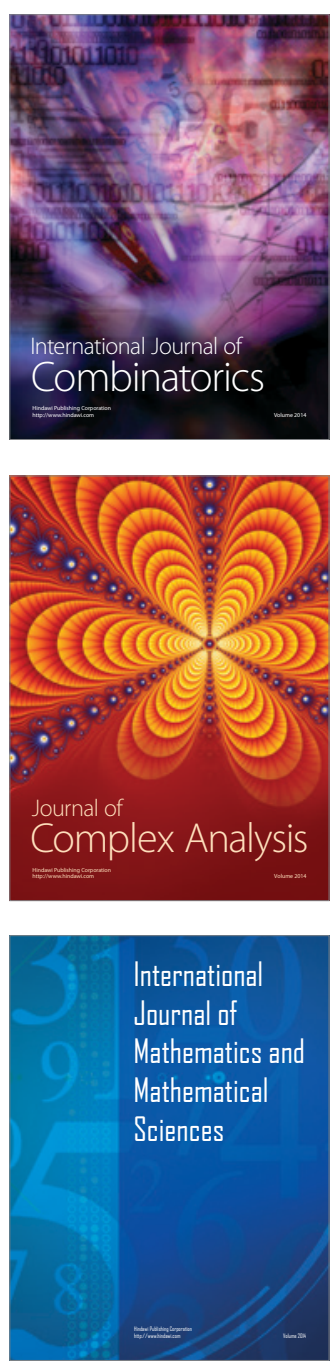
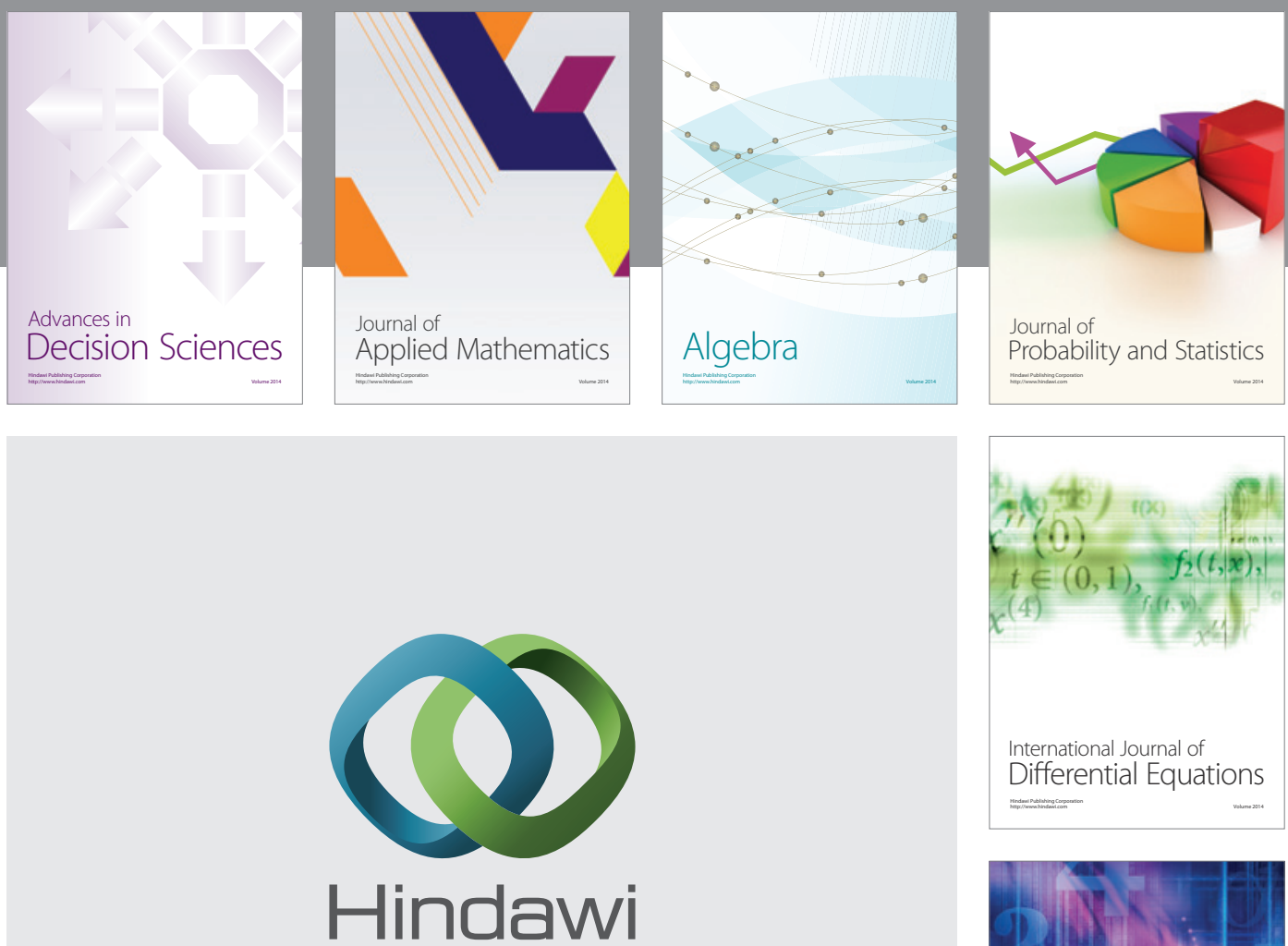

Submit your manuscripts at http://www.hindawi.com
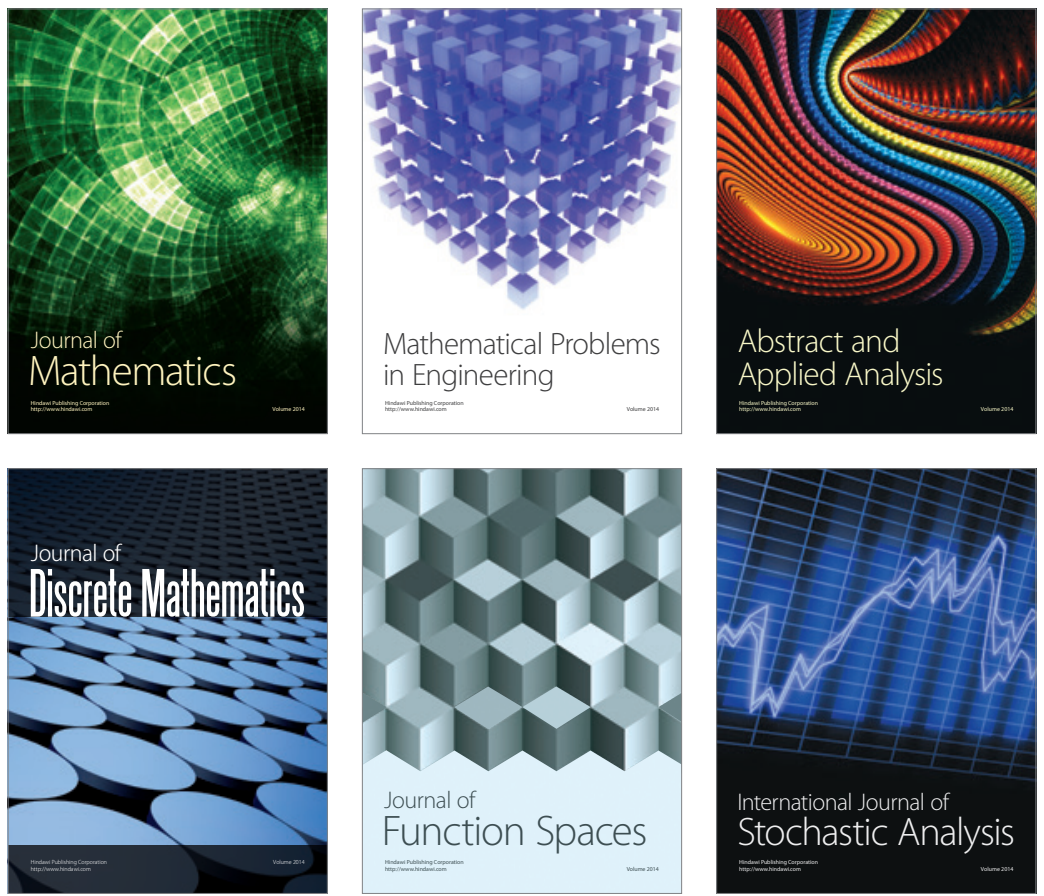

Journal of

Function Spaces

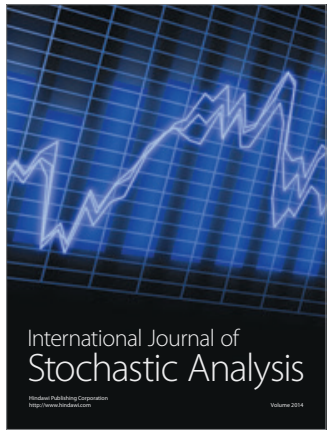

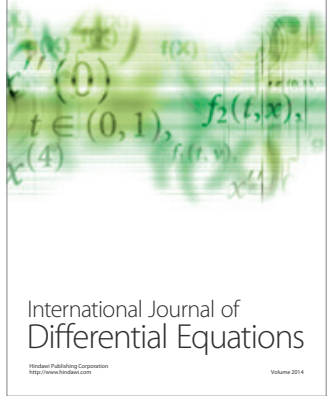
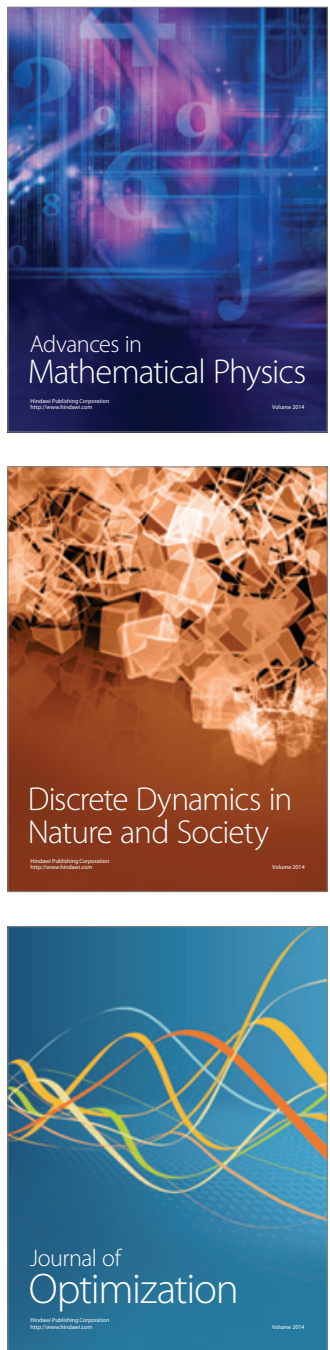